\title{
Erratum \\ Erratum to: Existence of Quasipattern Solutions of the Swift-Hohenberg Equation
}

\author{
Boele BraAksma, Gérard Iooss \& Laurent Stolovitch
}

\author{
Communicated by P. RABINOWITZ
}

\section{Erratum to: Arch. Rational Mech. Anal. (2013) 209:255-285 DOI 10.1007/s00205-013-0627-7}

We inform the readers that there is a mistake in the proof of our main theorem. We deeply thank Bob Rink who noticed a serious gap in the proof between Lemma 14 and Lemma 15. This computation of the perturbed eigenvalues is only valid for $\epsilon$ much smaller than all the scalar products $\mathbf{k}^{\prime} . \mathbf{k}_{j}, j=1, \ldots, 2 q$, which is not always the case here. So, additional work is needed to prove the existence theorem.

\author{
Johann Bernoulli Institute \\ University of Groningen \\ P.O. Box 407, 9700 AK Groningen, The Netherlands. \\ e-mail: B.L.J.Braaksma@rug.nl \\ and \\ Institut Universitaire de France-Laboratoire J.-A. Dieudonné U.M.R. 7351, \\ Université de Nice-Sophia Antipolis, \\ Parc Valrose, 06108 Nice Cedex 02, France. \\ e-mail: gerard.iooss@unice.fr \\ and \\ CNRS-Laboratoire J.-A. Dieudonné U.M.R. 7351 \\ Université de Nice-Sophia Antipolis, \\ Parc Valrose, 06108 Nice Cedex 02, \\ France. \\ e-mail: stolo@unice.fr
}

(Accepted November 20, 2013)

Published online December 18, 2013 - @ Springer-Verlag Berlin Heidelberg (2013)

The online version of the original article can be found under doi:10.1007/s00205-0130627-7. 